\title{
Comparison of three left atrial appendage occlusion devices for stroke prevention in patients with non-valvular atrial fibrillation: a single-centre seven-year experience with WATCHMAN, AMPLATZER Cardiac Plug/Amulet, LAmbre
}

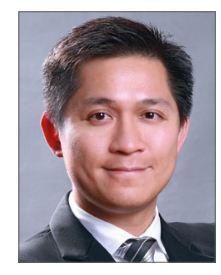

Gary SH. Cheung ${ }^{1}$, MBBS; Kent CY. So ${ }^{1}, \mathrm{MBChB}$; Christy KY. Chan ${ }^{1}$, MSc;

Anna KY. Chan ${ }^{1}, \mathrm{MBChB}$; Alex PW. Lee ${ }^{1}$, MBChB, MD; Yat-yin Lam², MBBS, MD, FACC;

Bryan P. Yan ${ }^{1 *}$, MBBS, MD, FACC

1. Division of Cardiology, Department of Medicine and Therapeutics, Faculty of Medicine, The Chinese University of Hong Kong and Prince of Wales Hospital, Hong Kong SAR, China; 2. Center Medical, Suite 1201, Central Building, Central, Hong Kong

SAR, China

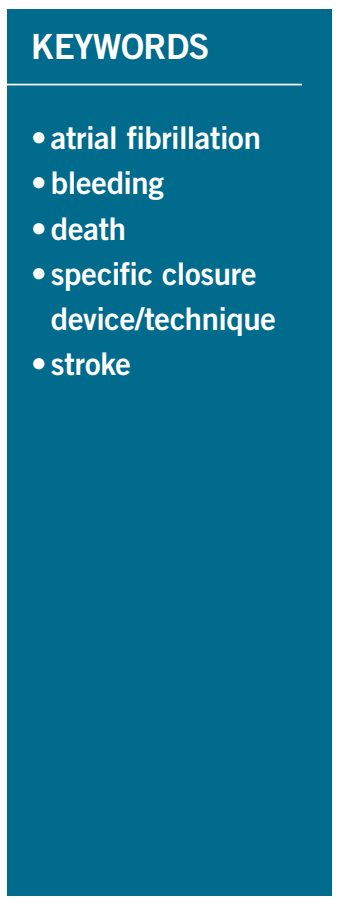

\section{Abstract}

Aims: We aimed to compare long-term "real-world" outcomes of three left atrial appendage occlusion (LAAO) devices for stroke prevention in a Chinese population with non-valvular atrial fibrillation (NVAF).

Methods and results: Consecutive patients who underwent LAAO from June 2009 to October 2016 at a university-affiliated hospital were retrospectively analysed. In-hospital and major adverse events (MAE) including mortality, stroke and major bleeding rates were compared by LAAO device. One hundred and sixty-one (161) patients (mean age $71.4 \pm 8.2$ years; $67.7 \%$ male) with mean $\mathrm{CHA}_{2} \mathrm{DS}_{2}$-VASc score of $4.1 \pm 1.6$ and HAS-BLED score of $2.9 \pm 1.1$ underwent 162 LAAO procedures, of which $47.5 \%$ ( $n=77), 41.4 \%$ $(\mathrm{n}=67)$ and $11.1 \%(\mathrm{n}=18)$ were AMPLATZER Cardiac Plug (ACP)/Amulet, WATCHMAN and LAmbre, respectively. The procedural success rate was $97.5 \%(158 / 162)$. The in-hospital adverse event rate was $7.4 \%(12 / 162)$ and comparable among devices ( $\mathrm{p}=\mathrm{NS})$. Mean follow-up duration was $28.3 \pm 24.4$ months (373 patient-years). There were no significant differences in long-term MAE rates among devices ( $\mathrm{p}=\mathrm{NS}$ ). Observed annual ischaemic stroke $(1.1 \%$ vs. $5.1 \%, \mathrm{p}<0.001)$ and major bleeding rates $(2.7 \%$ vs. $4.5 \%$, $\mathrm{p}=\mathrm{NS}$ ) were lower compared with the predicted rates, respectively.

Conclusions: The WATCHMAN, ACP/Amulet and LAmbre LAAO devices demonstrated similar longterm safety and efficacy in prevention of ischaemic stroke and major bleeding in patients with NVAF.

*Corresponding author: 9/F, Division of Cardiology, Department of Medicine and Therapeutics, Clinical Sciences Building, Prince of Wales Hospital, Shatin, N.T., Hong Kong SAR, China.E-mail: bryan.yan@cuhk.edu.hk 


\section{Abbreviations}

ACP
NVAF
CHA $_{2}$ DS $_{2}$-VASC
score

AMPLATZER Cardiac Plug

non-valvular atrial fibrillation

congestive heart failure, hypertension, age,

diabetes mellitus, previous stroke/transient

ischaemic attack, female sex, and vascular

disease score

HAS-BLED

score

hypertension, abnormal renal/liver function,

stroke, bleeding history or predisposition, labile

INR, elderly, drugs/alcohol concomitantly score

INR

LAAO

MAE

NOAC

NVAF

OAC

RRR

TEE

TIA

TTR

left atrial appendage occlusion

major adverse events

non-vitamin $\mathrm{K}$ oral anticoagulants

non-valvular atrial fibrillation

oral anticoagulation

relative risk reduction

transoesophageal echocardiography

transient ischaemic attack

time in therapeutic range

\section{Introduction}

The risk of ischaemic stroke in non-valvular atrial fibrillation (NVAF) patients is fivefold higher compared to the general population, accounting for about $15 \%$ of all strokes ${ }^{1}$. Oral anticoagulation (OAC) therapy with either warfarin or non-vitamin $\mathrm{K}$ oral anticoagulants (NOAC) is the standard treatment for stroke prevention in patients with NVAF at increased risk of stroke. Although effective in preventing stroke, OAC is associated with risk of bleeding and poor long-term drug compliance ${ }^{2}$. In a recent study of 1,461 Chinese patients with NVAF, $44.4 \%$ of patients discontinued warfarin within one year and $57.6 \%$ by two years after initiation of therapy ${ }^{3}$. In another study, $83.7 \%$ of Chinese patients on warfarin therapy failed to achieve $>65 \%$ time in therapeutic range (TTR) 4 .

Among patients who do not tolerate OAC, percutaneous left atrial appendage occlusion (LAAO) has emerged as an alternative treatment option for stroke prevention. The main advantages of LAAO over OAC include lower risk of major bleeding ${ }^{5}$ and issues with blood monitoring and drug compliance. Currently, the WATCHMANTM (Boston Scientific, Marlborough, MA, USA) and the AMPLATZER ${ }^{\text {TM }}$ Cardiac Plug (ACP) or later Amulet (St. Jude Medical, St. Paul, MN, USA) are the most commonly used commercially available LAAO devices. The LAmbre (Lifetech Scientific, Shenzhen, China) is a relatively new device which has recently received CE mark approval ${ }^{6}$.

In most published data on percutaneous LAAO, Asian patients consisted of $<1 \%$ of cases $^{7,8}$. In this study, we aimed to evaluate the safety and efficacy of different LAAO devices in Chinese NVAF patients for stroke prevention.

\section{Editorial, see page 15}

\section{Methods}

Consecutive NVAF patients who underwent LAAO at the Prince of Wales Hospital in Hong Kong were enrolled between June

2009 and October 2016. Data including baseline clinical and procedural characteristics, $\mathrm{CHA}_{2} \mathrm{DS}_{2}$-VASc and HAS-BLED scores, indication for LAAO, and follow-up clinical and transoesophageal echocardiography (TEE) results were recorded. The study was approved by the Joint Chinese University of Hong Kong - New Territories East Cluster Clinical Research Ethics Committee.

Indications for LAAO included NVAF (permanent, persistent, or paroxysmal) with a $\mathrm{CHA}_{2} \mathrm{DS}_{2}-\mathrm{VASc}$ score $\geq 2$ and one of the following: (i) history of bleeding; (ii) high risk of bleeding defined by HAS-BLED score $\geq 3$; (iii) labile international normalised ratio (INR) on warfarin; (iv) non-compliance with OAC therapy; and (v) allergy or side effects from OAC therapy.

\section{DEVICE AND IMPLANTING PROCEDURE}

Four LAAO devices were used in our institution. The WATCHMAN device has been available since October 2009, while the ACP was available from June 2009 and then the Amulet device from February 2015 onwards. The LAmbre device has been used since April 2014. The choice of device was at the discretion of the operator.

TEE was performed prior to LAAO procedure to rule out LAA thrombus and to measure LAA dimensions. After transseptal puncture, patients were heparinised to achieve an activated clotting time of 250-300 seconds throughout the procedure. A selective LAA angiogram was performed using a pigtail catheter. The device size was selected based on both angiographic and TEE measurements. Device stability, positioning, compression, and peri-device leaks were thoroughly assessed on TEE and angiography before final release of the device.

Chest radiography and transthoracic echocardiography were performed before discharge. Follow-up TEE was mandatory for all patients on day $45 \pm 7$ after the procedure. Lifelong aspirin ( $80 \mathrm{mg}$ daily) was prescribed for all patients. Clopidogrel $(75 \mathrm{mg}$ ) was prescribed for six months for the ACP/Amulet and LAmbre devices. For the WATCHMAN device, warfarin was administered for at least 45 days until mandatory follow-up TEE, and warfarin was discontinued if peri-device flow was $<5 \mathrm{~mm}$ on TEE and replaced with clopidogrel until six months after implant.

\section{DEFINITIONS}

Technical success was defined as successful implantation of the LAAO device without significant residual leak on TEE at the end of the procedure (i.e., $\leq 5 \mathrm{~mm}$ for all devices) 9 .

In-hospital adverse events were defined according to the Valve Academic Research Consortium-2 (VARC-2) criteria ${ }^{10}$ as any adverse events occurring post procedure and prior to discharge, including death, stroke, transient ischaemic attack (TIA), systemic embolisation, device embolisation, significant pericardial effusion or cardiac tamponade, myocardial infarction, major bleeding (requiring intervention or transfusion), and major vascular complications (requiring percutaneous or surgical intervention). Peri-device leak was considered significant if the colour Doppler jet width was $>5 \mathrm{~mm}$ for all devices on TEE 9 . Follow-up major 
adverse events (MAE), a composite of death, ischaemic or haemorrhagic stroke and TIA, or major bleeding, were recorded.

The actual event rates of stroke and bleeding at follow-up were compared with the predicted event rates by $\mathrm{CHA}_{2} \mathrm{DS}_{2}$-VASc and HAS-BLED scores, respectively. The average annual risks of stroke and bleeding for the study cohort were calculated. The actual $\%$ event rate was the total number of events divided by the total number of patient-years of follow-up and then multiplied by 100. Risk reduction was calculated using the formula: (estimated $\%$ event rate - actual $\%$ event rate)/ estimated $\%$ event rate ${ }^{11}$.

\section{STATISTICAL ANALYSIS}

Numerical results were presented as mean \pm SD or median (interquartile range $[\mathrm{IQR}])$. One-way ANOVA and Kruskal-Wallis tests were performed to compare means and medians among groups. Categorical results were presented as percentages and compared using the chi-square test or Fisher's exact test. Kaplan-Meier event-free survival curves were estimated, and differences were compared using the log-rank test. All statistical analyses were performed using SPSS statistical software, Version 22.0 (IBM Corp., Armonk, NY, USA). All analyses were two-tailed and p-values $<0.05$ were considered statistically significant.

\section{Results}

A total of 161 patients underwent 162 procedures. Baseline characteristics are summarised in Table 1. Mean patient age was $71.4 \pm 8.2$ years, $67.7 \%$ were male and more than one third had a history of stroke (38.5\%). The mean $\mathrm{CHA}_{2} \mathrm{DS}_{2}$-VASc score was $4.1 \pm 1.6$ and HAS-BLED score $2.9 \pm 1.1$. There were no significant differences in baseline characteristics among patients receiving different LAAO devices (all $\mathrm{p}=\mathrm{NS}$ ). The most common indication for LAAO was non-compliance to OAC therapy (34.2\%) followed by history of bleeding (31.7\%), high risk of bleeding (14.9\%) and labile INR on warfarin therapy (12.4\%). Non-compliance to OAC therapy included patients with poor drug compliance or refusal to continue OAC lifelong.

\section{PROCEDURE}

One patient underwent two LAAO procedures on different dates, resulting in 162 procedures. Of 162 procedures, 47.5\% ( $n=77)$, $41.4 \%(n=67)$ and $11.1 \%(n=18)$ were ACP/Amulet, WATCHMAN and LAmbre, respectively. Procedural findings are summarised in Table 1. Mean procedural time using the WATCHMAN, ACP/Amulet and LAmbre devices was $91.6 \pm 23.4$ minutes, $102.9 \pm 31.2$ minutes, and $89.1 \pm 23.3$ minutes, respectively $(\mathrm{p}=0.03)$. The median fluoroscopy time was 11 minutes (IQR 8-17.0) for WATCHMAN, 17 minutes (IQR 12-22) for ACP/Amulet, and 12 minutes (IQR 9-14.5) for the LAmbre device $(\mathrm{p}=<0.001)$.

The overall technical success rate was $97.5 \%$ (158/162). One patient failed the initial procedure due to ACP device embolisation, which was successfully retrieved with a snare. He subsequently underwent a successful second LAAO procedure with the WATCHMAN device. Three (two WATCHMAN and one ACP) procedures failed the stability test despite upsizing to the largest respective devices.

\section{IN-HOSPITAL EVENTS}

In-hospital adverse events occurred in 7.4\% (12/162) of cases (Table 2). There was a non-procedure-related death due to peritoneal dialysis peritonitis on day 15. Cardiac tamponade occurred in five patients (3.1\%); in four cases this required open surgical repair. There were three cases (1.9\%) of major bleeding from oesophageal laceration by the TEE probe. In one case the patient developed chest pain with acute ST elevation during the procedure; immediate coronary angiography revealed a critical stenosis of the left anterior descending artery which was successfully treated by angioplasty and stented without an associated rise in cardiac enzymes. There was one case $(0.6 \%)$ of a major femoral access-site complication which required endovascular intervention. The 30-day MAE rates were the same as the in-hospital MAE rates.

\section{TEE FOLLOW-UP}

Post-procedural TEE was performed in $94.3 \%$ of successful cases (149/158) (Table 3). Eight patients refused and one died before scheduled TEE. Significant peri-device leak was observed in $1.4 \%(1 / 71)$ of $\mathrm{ACP} /$ Amulet cases, but none with LAmbre or WATCHMAN devices. OAC therapy cessation at 45 days in the WATCHMAN group was $93.8 \%(61 / 65)$. Device thrombus was detected in five $(3.4 \%)$ patients by TEE (four WATCHMAN and one ACP). Clopidogrel was changed to OAC therapy in the patient with ACP device thrombus, and OAC therapy was extended for three months in the WATCHMAN patients. Follow-up TEE after three months showed complete resolution of thrombus in three cases (two WATCHMAN and one ACP) and persistent thrombus on two WATCHMAN devices. Long-term OAC therapy was continued for these two WATCHMAN patients.

\section{LONG-TERM FOLLOW-UP}

The mean follow-up duration was $28.3 \pm 24.4$ months and a total of 373 patient-years (Table 2). There were 14 deaths (8.9\%); however, none was related to the LAAO procedure (causes and time of death are shown in Table 4). Six strokes, including four ischaemic and two haemorrhagic strokes, occurred during follow-up.

The estimated annual risk of ischaemic stroke based on $\mathrm{CHA}_{2} \mathrm{DS}_{2}-$ VASc score was $5.1 \%$ compared to the observed rate of $1.1 \%$ (four events $/ 373$ patient-years). This translated to a $79.2 \%$ relative risk reduction $(R R R)$ in ischaemic stroke $(\mathrm{p}<0.001)$ (Figure 1). There were seven cases of major bleeding events during post-discharge follow-up. All bleeding events in the WATCHMAN group occurred during the period after stopping OAC. The observed annual major bleeding rate was $2.7 \%$ (10 events/373 patient-years) compared to a $4.5 \%$ predicted rate of annual major bleeding based on HASBLED score. This translated to a $39.9 \%$ RRR in major bleeding but was not statistically significant $(\mathrm{p}=0.10)$ (Figure 1 ). There were no significant differences in MAE-free survival rates among devices during follow-up (log-rank $\mathrm{p}=0.89$ ). 
Table 1. Patient characteristics and procedural findings.

\begin{tabular}{|c|c|c|c|c|c|c|}
\hline \multicolumn{2}{|c|}{ Patient characteristics } & $\underset{(n=66)}{\text { WATCHMAN }}$ & $\underset{(n=77)}{\text { ACP/Amulet }}$ & $\begin{array}{l}\text { LAmbre } \\
(n=18)\end{array}$ & $\begin{array}{c}\text { Total } \\
(n=161)\end{array}$ & $p$-value \\
\hline \multicolumn{2}{|l|}{ Age (years) } & $72.6 \pm 7.9$ & $71 \pm 8.6$ & $68.7 \pm 7$ & $71.4 \pm 8.2$ & 0.16 \\
\hline \multicolumn{2}{|l|}{ Male } & $45(68.2)$ & $50(64.9)$ & $14(77.8)$ & $109(67.7)$ & 0.57 \\
\hline \multicolumn{2}{|l|}{ Weight (kg) } & $65 \pm 10.3$ & $65.2 \pm 11.8$ & $69.1 \pm 11.1$ & $65.5 \pm 11.1$ & 0.35 \\
\hline \multicolumn{2}{|c|}{ Body mass index $\left(\mathrm{kg} / \mathrm{m}^{2}\right)$} & $25.2 \pm 2.9$ & $24.9 \pm 3.9$ & $25.7 \pm 3.8$ & $25.1 \pm 3.5$ & 0.70 \\
\hline \multicolumn{7}{|l|}{ Medical history } \\
\hline \multicolumn{2}{|l|}{ Prior heart failure } & $9(13.6)$ & $15(19.5)$ & $7(38.9)$ & $31(19.3)$ & 0.05 \\
\hline \multicolumn{2}{|l|}{ Hypertension } & $52(78.8)$ & $59(76.6)$ & $12(66.7)$ & $123(76.4)$ & 0.56 \\
\hline \multicolumn{2}{|l|}{ Diabetes mellitus } & $31(47.0)$ & $31(40.3)$ & $10(55.6)$ & $72(44.7)$ & 0.45 \\
\hline \multicolumn{2}{|l|}{ Previous stroke/TIA } & $28(42.4)$ & $26(33.8)$ & $8(44.4)$ & $62(38.5)$ & 0.49 \\
\hline \multicolumn{2}{|c|}{ Cardiovascular disease/peripheral arterial disease } & $8(12.1)$ & $12(15.6)$ & $2(11.1)$ & $22(13.7)$ & 0.79 \\
\hline \multicolumn{2}{|c|}{ Prior myocardial infarction } & $12(18.2)$ & $13(16.9)$ & $2(11.1)$ & $27(16.8)$ & 0.78 \\
\hline \multicolumn{2}{|c|}{ Prior percutaneous coronary intervention/coronary artery bypass grafting } & $13(19.7)$ & $22(28.6)$ & $3(16.7)$ & $38(23.6)$ & 0.35 \\
\hline \multicolumn{2}{|c|}{ Pacemaker implanted } & $8(12.1)$ & $18(23.4)$ & $2(11.1)$ & $28(17.4)$ & 0.16 \\
\hline \multicolumn{2}{|l|}{ History of bleeding } & $26(39.4)$ & $31(40.3)$ & $6(33.3)$ & $63(39.1)$ & 0.86 \\
\hline \multicolumn{2}{|c|}{$\mathrm{CHA}_{2} \mathrm{DS}_{2}$-VASc score (continuous) } & $4.3 \pm 1.4$ & $3.9 \pm 1.7$ & $3.8 \pm 1.4$ & $4.1 \pm 1.6$ & 0.37 \\
\hline \multirow{10}{*}{$\begin{array}{l}\mathrm{CHA}_{2} \mathrm{DS}_{2} \text {-VASc } \\
\text { score (categorical) }\end{array}$} & 0 & $0(0)$ & $0(0)$ & $0(0)$ & $0(0)$ & \multirow{10}{*}{0.45} \\
\hline & 1 & $1(1.5)$ & $6(7.8)$ & $0(0)$ & $7(4.3)$ & \\
\hline & 2 & $7(10.6)$ & $13(16.9)$ & $4(22.2)$ & $24(14.9)$ & \\
\hline & 3 & $10(15.2)$ & $11(14.3)$ & $3(16.7)$ & $24(14.9)$ & \\
\hline & 4 & $21(31.8)$ & $17(22.1)$ & 7 (38.9) & $45(28.0)$ & \\
\hline & 5 & $16(24.2)$ & $13(16.9)$ & $2(11.1)$ & $31(19.3)$ & \\
\hline & 6 & $6(9.1)$ & $14(18.2)$ & $1(5.6)$ & $21(13.0)$ & \\
\hline & 7 & $4(6.1)$ & $2(2.6)$ & $1(5.6)$ & $7(4.3)$ & \\
\hline & 8 & $1(1.5)$ & $1(1.3)$ & $0(0)$ & $2(1.2)$ & \\
\hline & 9 & $0(0)$ & $0(0)$ & $0(0)$ & $0(0)$ & \\
\hline \multicolumn{2}{|c|}{ HAS-BLED score (continuous) } & $3.1 \pm 1$ & $2.7 \pm 1.1$ & $2.8 \pm 0.9$ & $2.9 \pm 1.1$ & 0.12 \\
\hline \multirow{8}{*}{$\begin{array}{l}\text { HAS-BLED score } \\
\text { (categorical) }\end{array}$} & 0 & $1(1.5)$ & $0(0)$ & $0(0)$ & $1(0.6)$ & \\
\hline & 1 & $3(4.5)$ & $10(13)$ & $2(11.1)$ & $15(9.3)$ & \\
\hline & 2 & $10(15.2)$ & $24(31.2)$ & $3(16.7)$ & $37(23.0)$ & \\
\hline & 3 & $33(50.0)$ & $24(31.2)$ & $9(50.0)$ & $66(41.0)$ & 0.29 \\
\hline & 4 & $14(21.2)$ & $16(20.8)$ & $4(22.2)$ & $34(21.1)$ & $0 . \angle 9$ \\
\hline & 5 & $4(6.1)$ & $2(2.6)$ & $0(0)$ & $6(3.7)$ & \\
\hline & 6 & $1(1.5)$ & $1(1.3)$ & $0(0)$ & $2(1.2)$ & \\
\hline & $\geq 7$ & $0(0)$ & $0(0)$ & $0(0)$ & $0(0)$ & \\
\hline Atrial fibrillation & Paroxysmal & $19(28.8)$ & $23(29.9)$ & $5(27.8)$ & $47(29.2)$ & 0.98 \\
\hline & Permanent & $47(71.2)$ & $54(70.1)$ & $13(72.2)$ & $114(70.8)$ & \\
\hline Indications for left & Non-compliance to OAC/NOAC & $21(31.8)$ & $28(36.4)$ & $6(33.3)$ & $55(34.2)$ & \\
\hline atrıal appendage & Prior bleeding including previous intracranial bleeding & $20(30.3)$ & $26(33.8)$ & $5(27.8)$ & $51(31.7)$ & \\
\hline & High risk of bleeding & $14(21.2)$ & $8(10.4)$ & $2(11.1)$ & $24(14.9)$ & 0.21 \\
\hline & Labile INR & $9(13.6)$ & $8(10.4)$ & $3(16.7)$ & $20(12.4)$ & \\
\hline & Allergic, or side effect after OAC/NOAC & $0(0)$ & $7(9.1)$ & $1(5.6)$ & $8(5.0)$ & \\
\hline & Stroke on OAC/NOAC & $2(3)$ & $0(0)$ & $1(5.6)$ & $3(1.9)$ & \\
\hline & Procedural findings & $\underset{(n=67)}{\text { WATCHMAN }}$ & $\underset{(n=77)}{\text { ACP/Amulet }}$ & $\begin{array}{l}\text { LAmbre } \\
(\mathrm{n}=18)\end{array}$ & $\begin{array}{c}\text { Total } \\
(n=162)\end{array}$ & $p$-value \\
\hline Anaesthesia & General anaesthesia & $23(34.3)$ & $31(40.3)$ & $0(0)$ & $54(33.3)$ & \\
\hline & Local anaesthesia & $0(0)$ & $3(3.9)$ & $17(94.4)$ & $20(12.3)$ & $<0.001$ \\
\hline & Monitored anaesthetic care & $44(65.7)$ & $43(55.8)$ & $1(5.6)$ & $88(54.3)$ & \\
\hline Procedural time, mi & nutes & $91.6 \pm 23.4$ & $102.9 \pm 31.2$ & $89.1 \pm 23.3$ & $96.6 \pm 27.8$ & 0.03 \\
\hline Fluoroscopy time, m & inutes & $11(8-17)$ & $17(12-22)$ & $12(9-14.5)$ & $14(10-19)$ & $<0.001$ \\
\hline Length of stay, days & & $3(2-4)$ & $3(2-4)$ & $2(2-2.3)$ & $3(2-4)$ & 0.08 \\
\hline Procedural success & & $65(97.0)$ & $75(97.4)$ & $18(100)$ & $158(97.5)$ & 0.77 \\
\hline
\end{tabular}


Table 2. In-hospital events of patients undergoing LAAO and long-term follow-up outcomes of successfully implanted patients.

\begin{tabular}{|c|c|c|c|c|c|}
\hline & $\begin{array}{c}\text { WATCH- } \\
\text { MAN } \\
(n=67)\end{array}$ & $\begin{array}{c}\text { ACP/ } \\
\text { Amulet } \\
(n=77)\end{array}$ & $\begin{array}{c}\text { LAmbre } \\
(\mathrm{n}=18)\end{array}$ & $\begin{array}{c}\text { Total } \\
(n=162)\end{array}$ & $p$-value ${ }^{a}$ \\
\hline \multicolumn{6}{|l|}{ In-hospital events } \\
\hline Death & $1(1.5)$ & $0(0)$ & $0(0)$ & $1(0.6)$ & 0.47 \\
\hline Cardiac death & $0(0)$ & $0(0)$ & $0(0)$ & $0(0)$ & NA \\
\hline Non-cardiac death & $1(1.5)$ & $0(0)$ & $0(0)$ & $1(0.6)$ & 0.47 \\
\hline Stroke/TIA & $0(0)$ & $0(0)$ & $0(0)$ & $0(0)$ & NA \\
\hline Device embolisation & $0(0)$ & $1(1.3)$ & $0(0)$ & $1(0.6)$ & 1.00 \\
\hline Systemic embolism & $0(0)$ & $0(0)$ & $0(0)$ & $0(0)$ & NA \\
\hline Cardiac tamponade & 2 (3) & $3(3.9)$ & $0(0)$ & $5(3.1)$ & 1.00 \\
\hline Major bleeding & $0(0)$ & $3(3.9)$ & $0(0)$ & $3(1.9)$ & 0.25 \\
\hline Myocardial infarction & $1(1.5)$ & $0(0)$ & $0(0)$ & $1(0.6)$ & 0.47 \\
\hline $\begin{array}{l}\text { Major vascular complications } \\
\text { (requiring endovascular } \\
\text { intervention) }\end{array}$ & $0(0)$ & $1(1.3)$ & $0(0)$ & $1(0.6)$ & 1.00 \\
\hline Total & $4(6.0)$ & $8(10.4)$ & $0(0)$ & $12(7.4)$ & 0.34 \\
\hline Long-term follow-up (FU) & $\begin{array}{c}\text { WATCH- } \\
\text { MAN } \\
(n=65)\end{array}$ & $\begin{array}{c}\text { ACP/ } \\
\text { Amulet } \\
(n=75)\end{array}$ & $\begin{array}{c}\text { LAmbre } \\
(\mathrm{n}=18)\end{array}$ & $\begin{array}{c}\text { Total } \\
(n=158)\end{array}$ & $p$-value ${ }^{b}$ \\
\hline FU months & $27.5 \pm 24.2$ & $30.1 \pm 27$ & $23.5 \pm 8.3$ & $28.3 \pm 24.4$ & 0.55 \\
\hline FU, total patient-years & 149 & 189 & 35 & 373 & NA \\
\hline \multicolumn{6}{|l|}{ Risk scores } \\
\hline $\mathrm{CHA}_{2} \mathrm{DS}_{2}$-VASc score & $4.2 \pm 1.5$ & $4.0 \pm 1.7$ & $3.8 \pm 1.4$ & $4.1 \pm 1.6$ & 0.52 \\
\hline $\begin{array}{l}\text { Estimated annual risk of } \\
\text { stroke, based on } \\
\text { CHA }_{2} \text { SS }_{2} \text {-VASc score, \% }\end{array}$ & $5.25 \pm 2.64$ & $5.22 \pm 3.02$ & $4.40 \pm 2.32$ & $5.14 \pm 2.79$ & 0.50 \\
\hline HAS-BLED score & $3.1 \pm 1$ & $2.7 \pm 1.1$ & $2.8 \pm 0.9$ & $2.9 \pm 1.1$ & 0.15 \\
\hline $\begin{array}{l}\text { Estimated annual risk of } \\
\text { major bleeding, based on } \\
\text { HAS-BLED score, \% }\end{array}$ & $4.91 \pm 3.15$ & $4.12 \pm 3.07$ & $4.23 \pm 2.65$ & $4.46 \pm 3.06$ & 0.31 \\
\hline \multicolumn{6}{|l|}{ Death } \\
\hline In-hospital & $1(1.5)$ & $0(0)$ & $0(0)$ & $1(0.6)$ & 0.49 \\
\hline Cardiac death & $0(0)$ & $0(0)$ & $0(0)$ & $0(0)$ & NA \\
\hline Non-cardiac death & $1(1.5)$ & $0(0)$ & $0(0)$ & $1(0.6)$ & 0.49 \\
\hline Post-discharge & $4(6.2)$ & $7(9.3)$ & $3(16.7)$ & $14(8.9)$ & 0.37 \\
\hline Cardiac death & $1(1.5)$ & $1(1.3)$ & $1(5.6)$ & $3(1.9)$ & 0.48 \\
\hline Non-cardiac death & $3(4.6)$ & $6(8.0)$ & 2 (11.1) & $12(7.6)$ & 0.56 \\
\hline Total & $5(7.7)$ & $7(9.3)$ & $3(16.7)$ & $15(9.5)$ & 0.51 \\
\hline
\end{tabular}

\begin{tabular}{|c|c|c|c|c|c|}
\hline & $\begin{array}{c}\text { WATCH- } \\
\text { MAN } \\
(n=67)\end{array}$ & $\begin{array}{c}\text { ACPI } \\
\text { Amulet } \\
(\mathrm{n}=77)\end{array}$ & $\begin{array}{c}\text { LAmbre } \\
(\mathrm{n}=18)\end{array}$ & $\begin{array}{c}\text { Total } \\
(n=162)\end{array}$ & $p$-value ${ }^{a}$ \\
\hline \multicolumn{6}{|l|}{ Stroke/TIA } \\
\hline In-hospital & $0(0)$ & $0(0)$ & $0(0)$ & $0(0)$ & NA \\
\hline Ischaemic & $0(0)$ & $0(0)$ & $0(0)$ & $0(0)$ & NA \\
\hline Haemorrhagic & $0(0)$ & $0(0)$ & $0(0)$ & $0(0)$ & NA \\
\hline Post-discharge & $2(3.1)$ & $3(4.0)$ & $1(5.6)$ & $6(3.8)$ & 0.88 \\
\hline Ischaemic & $1(1.5)$ & $3(4.0)$ & $0(0)$ & $4(2.5)$ & 0.50 \\
\hline Haemorrhagic & $1(1.5)$ & $0(0)$ & $1(5.6)$ & $2(1.3)$ & 0.16 \\
\hline Total & $2(3.1)$ & $3(4.0)$ & $1(5.6)$ & $6(3.8)$ & 0.95 \\
\hline $\begin{array}{l}\text { Observed annual risk of } \\
\text { stroke, \% }\end{array}$ & $\begin{array}{c}0.67 \\
(1 / 149) \\
* 100\end{array}$ & $\begin{array}{c}1.59 \\
(3 / 189) \\
* 100\end{array}$ & $\begin{array}{c}0 \\
(0 / 35) \\
* 100\end{array}$ & $\begin{array}{c}1.07 \\
(4 / 373) \\
* 100\end{array}$ & 0.75 \\
\hline Stroke reduction, \% & $\begin{array}{c}87.2 \\
([5.25- \\
0.67] / 5.25) \\
* 100\end{array}$ & $\begin{array}{c}69.5 \\
([5.22- \\
1.59] / 5.22) \\
* 100\end{array}$ & $\begin{array}{c}100.0 \\
([4.40- \\
0] / 4.40) \\
* 100\end{array}$ & $\begin{array}{c}79.2 \\
([5.14- \\
1.07] / 5.14) \\
* 100\end{array}$ & $<0.001$ \\
\hline NNT & 22 & 28 & 23 & 25 & \\
\hline
\end{tabular}

\section{Major bleeding}

\begin{tabular}{|c|c|c|c|c|c|}
\hline In-hospital & $0(0)$ & $3(4.0)$ & $0(0)$ & $3(1.9)$ & 0.18 \\
\hline Post-discharge & $4(6.2)$ & $2(2.7)$ & $1(5.6)$ & $7(4.4)$ & 0.59 \\
\hline Total & $4(6.2)$ & $5(6.7)$ & $1(5.6)$ & $10(6.3)$ & 0.95 \\
\hline $\begin{array}{l}\text { Observed annual risk of } \\
\text { major bleeding, \% }\end{array}$ & $\begin{array}{c}2.68 \\
(4 / 149) \\
* 100\end{array}$ & $\begin{array}{c}2.65 \\
(5 / 189) \\
{ }^{*} 100\end{array}$ & $\begin{array}{c}2.86 \\
(1 / 35) \\
* 100\end{array}$ & $\begin{array}{c}2.68 \\
(10 / 373) \\
* 100\end{array}$ & 1.00 \\
\hline Major bleeding reduction, \% & $\begin{array}{c}45.4 \\
([4.91- \\
2.68] / 4.91) \\
* 100\end{array}$ & $\begin{array}{c}35.7 \\
([4.12- \\
2.65] / 4.12) \\
* 100\end{array}$ & $\begin{array}{c}32.4 \\
([4.23- \\
2.86] / 4.23) \\
* 100\end{array}$ & $\begin{array}{c}39.9 \\
([4.46- \\
2.68] / 4.46) \\
* 100\end{array}$ & 0.16 \\
\hline NNT & 45 & 68 & 73 & 57 & \\
\hline
\end{tabular}

\section{Major adverse events (MAE)}

\begin{tabular}{|l|c|c|l|l|l|}
\hline In-hospital & $1(1.5)$ & $3(4.0)$ & $0(0)$ & $4(2.5)$ & 0.50 \\
\hline Post-discharge & $9(13.8)$ & $10(13.3)$ & $4(22.2)$ & $23(14.6)$ & 0.62 \\
\hline Total & $10(15.4)$ & $13(17.3)$ & $4(22.2)$ & $27(17.1)$ & 0.89 \\
\hline
\end{tabular}

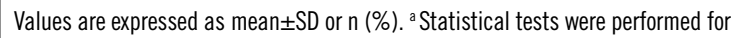
comparison between WATCHMAN and ACP/Amulet devices. ${ }^{\mathrm{b}}$ Statistical tests were performed for comparison between WATCHMAN, ACP/Amulet and LAmbre devices. ${ }^{\circ}$ A major adverse event (MAE) is defined as a composite of death, ischaemic or haemorrhagic stroke and TIA, or major bleeding. ACP: AMPLATZER Cardiac Plug; NNT: number needed to treat;

TIA: transient ischaemic attack

\section{Discussion}

The main finding of this study was that different LAAO devices comprising the WATCHMAN, ACP/Amulet and LAmbre were comparable in terms of long-term efficacy in reducing ischaemic stroke and avoiding major bleeding events in Chinese NVAF patients. To the best of our knowledge, this is the largest reported cohort of LAAO with the longest follow-up in a Chinese population and the first clinical experience with the LAmbre device.

Our procedural success rate $(97.5 \%)$ was comparable with other LAAO studies, with reported success rates from $90.9 \%$ to $98.5 \%{ }^{7,8,11,12}$. The safety profile of LAAO in our cohort also

compared favourably with these studies. Our in-hospital MAE rate was driven by the occurrence of cardiac tamponade ( $n=5 / 12 \mathrm{MAE}$ ), which occurred during the early phase of our LAAO programme. A similar learning curve has been reported with the WATCHMAN device where rates of cardiac tamponade decreased over time with increasing operator experience from $4 \%$ in PROTECT $\mathrm{AF}^{7}$ to $1.9 \%$ in PREVAIL $^{8}$ and $0.2 \%$ in EWOLUTION ${ }^{12}$. Furthermore, after two cases of guidewire perforation using the Amplatz Super Stiff ${ }^{\text {TM }}$ guidewire (Boston Scientific), our procedural protocol was revised and placement of the Super Stiff guidewire within the LAA was no longer allowed. No guidewire-related perforation or cardiac tamponade occurred after this change. 


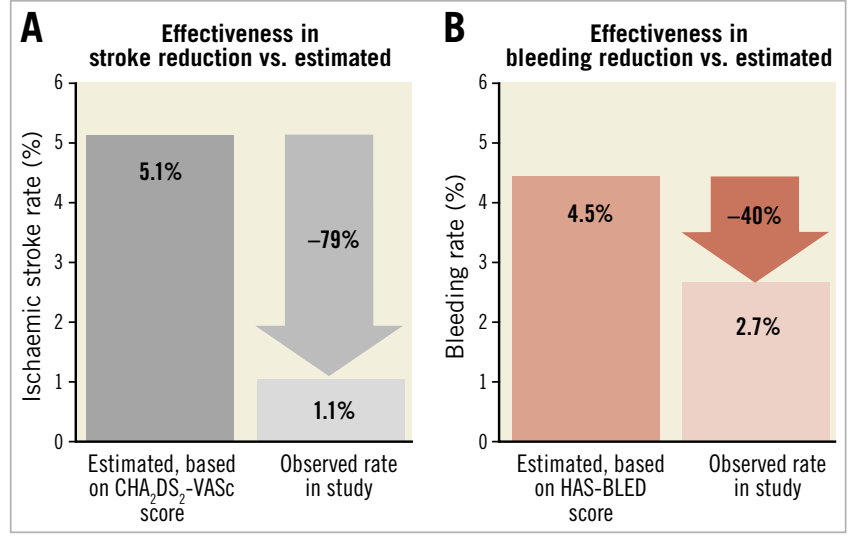

Figure 1. Effectiveness of LAAO in reduction of $(A)$ ischaemic stroke rate and $(B)$ major bleeding rate compared with estimated annual rates from $\mathrm{CHA}_{2} D S_{2}$-VASc score and HAS-BLED score, respectively.

When LAAO devices are recaptured and repositioned repeatedly, the reverse-facing stabilising hooks can cause damage and perforation to the LAA wall. Accurate preoperative device sizing is crucial to prevent this complication. Traditionally, device sizing is guided by TEE and fluoroscopy. Recently, we have incorporated $3 \mathrm{D}$ printed modelling of the LAA from CT images for preprocedural planning and device size selection ${ }^{13}$ to reduce inappropriate sizing and device manipulation during implantation.

Long-term compliance with warfarin treatment for stroke prevention is a major problem in Chinese patients with $\mathrm{NVAF}^{3}$. In our cohort of Chinese patients, non-compliance to OAC therapy (34.2\%) was the most common indication for LAAO, whereas previous bleeding and high bleeding risk were the major indications for LAAO in Caucasian populations ${ }^{11,12}$. LAAO obviates the need for long-term anticoagulation (except in a small number of patients with persistent device-related thrombus) and hence the risk of bleeding and also stroke from subtherapeutic INR. Although there are no direct comparisons between LAAO and NOAC, our group recently published a study using the Markov decision analytic model to compare the cost-effectiveness of LAAO with seven pharmacological strategies including aspirin alone, clopidogrel plus aspirin, warfarin, dabigatran (110 $\mathrm{mg}$ and $150 \mathrm{mg}$ ), apixaban and rivaroxaban from a Hong Kong healthcare provider perspective ${ }^{14}$. We found that LAAO was considered cost-effective compared to warfarin, with an incremental cost-effectiveness ratio (ICER) of USD 6,298 per quality-adjusted life years (QALY) gained; LAAO was considered dominant (i.e., less costly and more effective) compared to all three NOAC. Sensitivity analysis demonstrated that ICERs of LAAO remained favourable against NOAC in various

Table 3. Transoesophageal echocardiography (TEE) follow-up findings.

\begin{tabular}{|c|c|c|c|c|c|}
\hline & WATCHMAN $(n=61)$ & ACP/Amulet $(n=71)$ & LAmbre $(n=17)$ & Total $(n=149)$ & $p$-value \\
\hline Thrombus detection over device & $4(6.6)$ & $1(1.4)$ & $0(0)$ & $5(3.4)$ & 0.19 \\
\hline Leak at TEE follow-up 45 days & $20(32.8)$ & $13(18.3)$ & $8(47.1)$ & $41(27.5)$ & 0.03 \\
\hline Significant peri-device leak ${ }^{a}$ & $0(0)$ & $1(1.4)$ & $0(0)$ & $1(0.7)$ & 0.58 \\
\hline
\end{tabular}

Table 4. Characteristics of deceased patients.

\begin{tabular}{|c|c|c|c|c|c|c|c|}
\hline Patient & $\begin{array}{c}\text { Age } \\
\text { (years) }\end{array}$ & Sex & $\begin{array}{l}\mathrm{CHA}_{2} \mathrm{DS}_{2}^{-} \\
\text {VASc score }\end{array}$ & $\begin{array}{l}\text { HAS-BLED } \\
\text { score }\end{array}$ & Device & Cause of death & $\begin{array}{l}\text { Time between procedure } \\
\text { and death (months) }\end{array}$ \\
\hline 1 & 62 & $\mathrm{M}$ & 1 & 1 & ACP & Brain tumour & 20 \\
\hline 2 & 69 & M & 3 & 3 & ACP & Ischaemic stroke on warfarin & 76 \\
\hline 3 & 48 & M & 5 & 2 & ACP & Type A aortic dissection & 65 \\
\hline 4 & 81 & $\mathrm{M}$ & 4 & 2 & WATCHMAN & Acute renal failure & 84 \\
\hline 5 & 63 & $\mathrm{M}$ & 3 & 2 & $\mathrm{ACP}$ & Liver tumour & 39 \\
\hline 6 & 66 & M & 5 & 3 & WATCHMAN & Sudden collapse & 22 \\
\hline 7 & 83 & $\mathrm{~F}$ & 8 & 4 & WATCHMAN & End-stage renal failure & 17 \\
\hline 8 & 65 & M & 6 & 4 & ACP & Pneumonia & 24 \\
\hline 9 & 76 & $\mathrm{M}$ & 4 & 4 & WATCHMAN & Necrotising fasciitis & 16 \\
\hline 10 & 68 & $\mathrm{M}$ & 5 & 4 & LAmbre & Pneumonia & 4 \\
\hline 11 & 80 & M & 5 & 3 & LAmbre & Haemorrhagic stroke & 18 \\
\hline 12 & 65 & M & 3 & 2 & LAmbre & Unknown cause & 6 \\
\hline 13 & 80 & $\mathrm{~F}$ & 5 & 3 & ACP & Malignant duodenal obstruction & 6 \\
\hline 14 & 83 & $\mathrm{~F}$ & 7 & 6 & WATCHMAN & Peritoneal dialysis peritonitis & 1 \\
\hline 15 & 84 & M & 5 & 4 & Amulet & Pneumonia & 7 \\
\hline \multicolumn{8}{|c|}{ ACP: AMPLATZER Cardiac Plug } \\
\hline
\end{tabular}


$\mathrm{CHADS}_{2}$ scores, HAS-BLED scores, time horizons and LAAO costs. Therefore, LAAO could be considered a cost-effective option for stroke prophylaxis in Chinese patients with NVAF.

\section{Limitations}

The limitations of the study included a small sample size and being from a single centre. Nonetheless, this is the largest reported cohort of LAAO with the longest follow-up in Chinese patients with NVAF.

\section{Conclusions}

Percutaneous LAAO was a safe and effective treatment option for stroke prophylaxis in Chinese patients with NVAF. The WATCHMAN, ACP/Amulet and LAmbre LAAO devices were comparable in long-term safety and efficacy in stroke reduction and avoidance of major bleeding.

\section{Impact on daily practice}

The majority of published data concerning left atrial appendage occlusion (LAAO) have come from Western populations. This article provides "real-world" long-term data of LAAO in a Chinese population. We demonstrated that LAAO with three different devices is a safe and effective treatment strategy for stroke prophylaxis in Chinese patients with non-valvular atrial fibrillation who are not candidates for oral anticoagulation therapy.

\section{Conflict of interest statement}

G. Cheung is a clinical proctor for WATCHMAN and Amulet LAAO to Boston Scientific and St. Jude Medical, respectively. Y-y. Lam is a clinical proctor for LAmbre and Amulet LAAO to Lifetech and St. Jude Medical, respectively. The other authors have no conflicts of interest to declare.

\section{References}

1. Lin HJ, Wolf PA, Kelly-Hayes M, Beiser AS, Kase CS, Benjamin EJ, D'Agostino RB. Stroke severity in atrial fibrillation. The Framingham Study. Stroke. 1996;27:1760-4.

2. Hylek EM, Evans-Molina C, Shea C, Henault LE, Regan S. Major hemorrhage and tolerability of warfarin in the first year of therapy among elderly patients with atrial fibrillation. Circulation. 2007;115:2689-96.

3. Wang ZZ, Du X, Wang W, Tang RB, Luo JG, Li C, Chang SS, Liu XH, Sang CH, Yu RH, Long DY, Wu JH, Bai R, Liu N, Ruan YF, Dong JZ, Ma CS. Long-Term Persistence of Newly Initiated Warfarin Therapy in Chinese Patients With Nonvalvular Atrial Fibrillation. Circ Cardiovasc Qual Outcomes. 2016;9:380-7.

4. Li WH, Huang D, Chiang CE, Lau CP, Tse HF, Chan EW, Wong ICK, Lip GYH, Chan PH, Siu CW. Efficacy and safety of dabigatran, rivaroxaban, and warfarin for stroke prevention in Chinese patients with atrial fibrillation: the Hong Kong Atrial Fibrillation Project. Clin Cardiol. 2017;40:222-9.
5. Reddy VY, Sievert H, Halperin J, Doshi SK, Buchbinder M, Neuzil P, Huber K, Whisenant B, Kar S, Swarup V, Gordon N, Holmes D; PROTECT AF Steering Committee and Investigators. Percutaneous left atrial appendage closure vs warfarin for atrial fibrillation: a randomized clinical trial. JAMA. 2014;312:1988-98.

6. Lam YY. A new left atrial appendage occluder (Lifetech LAmbre Device) for stroke prevention in atrial fibrillation. Cardiovasc Revasc Med. 2013;14:134-6.

7. Holmes DR, Reddy VY, Turi ZG, Doshi SK, Sievert H, Buchbinder M, Mullin CM, Sick P; PROTECT AF Investigators. Percutaneous closure of the left atrial appendage versus warfarin therapy for prevention of stroke in patients with atrial fibrillation: a randomised non-inferiority trial. Lancet. 2009;374:534-42.

8. Holmes DR Jr, Kar S, Price MJ, Whisenant B, Sievert H, Doshi SK, Huber K, Reddy VY. Prospective randomized evaluation of the Watchman Left Atrial Appendage Closure device in patients with atrial fibrillation versus long-term warfarin therapy: the PREVAIL trial. J Am Coll Cardiol. 2014;64:1-12.

9. Tzikas A, Holmes DR Jr, Gafoor S, Ruiz CE, BlomströmLundqvist C, Diener HC, Cappato R, Kar S, Lee RJ, Byrne RA, Ibrahim R, Lakkireddy D, Soliman OI, Nabauer M, Schneider S, Brachmann J, Saver JL, Tiemann K, Sievert H, Camm AJ, Lewalter T. Percutaneous left atrial appendage occlusion: the Munich consensus document on definitions, endpoints, and data collection requirements for clinical studies. Europace. 2017;19:4-15.

10. Kappetein AP, Head SJ, Généreux P, Piazza N, van Mieghem NM, Blackstone EH, Brott TG, Cohen DJ, Cutlip DE, van Es GA, Hahn RT, Kirtane AJ, Krucoff MW, Kodali S, Mack MJ, Mehran R, Rodés-Cabau J, Vranckx P, Webb JG, Windecker S, Serruys PW, Leon MB. Updated standardized endpoint definitions for transcatheter aortic valve implantation: the Valve Academic Research Consortium-2 consensus document. J Am Coll Cardiol. 2012;60:1438-54.

11. Tzikas A, Shakir S, Gafoor S, Omran H, Berti S, Santoro G, Kefer J, Landmesser U, Nielsen-Kudsk JE, Cruz-Gonzalez I, Sievert H, Tichelbäcker T, Kanagaratnam P, Nietlispach F, Aminian A, Kasch F, Freixa X, Danna P, Rezzaghi M, Vermeersch P, Stock F, Stolcova M, Costa M, Ibrahim R, Schillinger W, Meier B, Park JW. Left atrial appendage occlusion for stroke prevention in atrial fibrillation: multicenter experience with the AMPLATZER Cardiac Plug. EuroIntervention. 2016;11:1170-9.

12. Kische S, Schmitz T, Stein KM, Bergmann MW; EWOLUTION investigators. Implant success and safety of left atrial appendage closure with the WATCHMAN device: periprocedural outcomes from the EWOLUTION registry. Eur Heart J. 2016;37:2465-74.

13. Fan Y, Kwok KW, Zhang Y, Cheung GS, Chan AK, Lee AP. Three-Dimensional Printing for Planning Occlusion Procedure for a Double-Lobed Left Atrial Appendage. Circ Cardiovasc Interv. 2016;9:e003561.

14. Lee VW, Tsai RB, Chow IH, Yan BP, Kaya MG, Park JW, Lam YY. Cost-effectiveness analysis of left atrial appendage occlusion compared with pharmacological strategies for stroke prevention in atrial fibrillation. BMC Cardiovasc Disord. 2016;16:167. 